\title{
Transparent functional oxide stretchable electronics: micro-tectonics enabled high strain electrodes
}

\author{
Philipp Gutruf ${ }^{1,2}$, Charan M Shah ${ }^{1}$, Sumeet Walia ${ }^{1}$, Hussein Nili ${ }^{1}$, Ahmad S Zoolfakar ${ }^{1}$, Christian Karnutsch ${ }^{2}$, \\ Kourosh Kalantar-zadeh ${ }^{1}$, Sharath Sriram ${ }^{1}$ and Madhu Bhaskaran ${ }^{1}$ \\ Fully transparent and flexible electronic substrates that incorporate functional materials are the precursors to realising next- \\ generation devices with sensing, self-powering and portable functionalities. Here, we demonstrate a universal process for \\ transferring planar, transparent functional oxide thin films on to elastomeric polydimethylsiloxane (PDMS) substrates. This \\ process overcomes the challenge of incorporating high-temperature-processed crystalline oxide materials with low-temperature \\ organic substrates. The functionality of the process is demonstrated using indium tin oxide (ITO) thin films to realise fully \\ transparent and flexible resistors. The ITO thin films on PDMS are shown to withstand uniaxial strains of $15 \%$, enabled by \\ microstructure tectonics. Furthermore, zinc oxide was transferred to display the versatility of this transfer process. Such a \\ ubiquitous process for the transfer of functional thin films to elastomeric substrates will pave the way for touch sensing and \\ energy harvesting for displays and electronics with flexible and transparent characteristics.
}

NPG Asia Materials (2013) 5, e62; doi:10.1038/am.2013.41; published online 13 September 2013

Keywords: flexible electronics; functional oxides; high-temperature oxides; ITO; PDMS; transparent materials; ZnO

\section{INTRODUCTION}

Novel micro- and nano-technology applications encompassing plasmonic devices, field effect transistors, light-emitting diodes, sensor networks, electromagnetic components, terahertz metamaterials, energy harvesters and displays are increasingly demonstrated on flexible substrates. ${ }^{1-7}$ These applications represent the building blocks of future flexible and transparent device technology incorporating complex circuitry and functionality. In integrating all the applications together to realise powerful and practical technology that is fully transparent, flexible and functional, two major challenges need to be overcome.

First, the flexible substrate should ideally be transparent and colourless. A variety of colourless materials such as polyethylene ${ }^{8}$ and polydimethylsiloxane (PDMS) ${ }^{1}$ and coloured materials such as polyimide (or Kapton) $)^{9}$ are widely used. The former can withstand only low processing temperatures $\left(<100^{\circ} \mathrm{C}\right)$, while the yellow-brown tinted polyimide can withstand up to $\sim 400^{\circ} \mathrm{C}$. The relevance of colourless substrates to flexible device technology is highlighted by the recent research focus on this area. ${ }^{10,11}$ Second, functional oxide materials that offer tailored properties need to be integrated. These functional oxides in the form of thin films can be transparent conductive oxides for electrical conduction, ferroelectrics for memories, piezoelectrics for energy harvesting, ${ }^{12}$ and semiconductors or dielectrics for high-performance transistors. Almost all high-performance functional oxides need to be crystalline and are deposited at high temperatures (ranging from 400 to $700^{\circ} \mathrm{C}$ ), ${ }^{13,14}$ ruling out direct deposition on to the polymeric flexible substrates. Although alternatives to functional oxides such as poly(3,4-ethylenedioxythiophene) poly(styrenesulfonate) exist, ${ }^{15}$ the integration in current microelectronic devices is not fully developed yet.

In offering a potential solution to these challenges, this work presents a universal technique for the incorporation of high-temperature-processed functional oxides with a flexible, elastomeric substrate. It utilises standard microfabrication and materials processing techniques, enabling features of large area fabrication and scalability. The substrate material chosen for this work is PDMS, which is widely used in flexible electronics and microfluidics. ${ }^{16,17}$ The technique is demonstrated with indium tin oxide (ITO), the extensively adopted transparent conductive oxide, as the functional layer allowing us to realise and demonstrate electronics that is transparent, flexible and stretchable. This demonstration also led to the determination of high uniaxial strain performance of ITO on PDMS resistor structures, enabled by micro-tectonics in the corrugated oxide thin film. Furthermore, zinc oxide $(\mathrm{ZnO})$ was chosen to demonstrate the ability to transfer a high-quality semiconducting oxide that is currently very demanded in research because of its suitability for a wide variety of applications. ${ }^{18}$ Unlike transfer printing methods, ${ }^{19}$ where the finished functional layers are picked and placed from a

${ }^{1}$ Functional Materials and Microsystems Research Group, School of Electrical and Computer Engineering, RMIT University, Melbourne, Victoria, Australia and ${ }^{2}$ Department of Electrical Engineering and Information Technology, Institute for Optofluidics and Integrated Nanophotonics, Karlsruhe University of Applied Sciences, Karlsruhe, Germany Correspondence: Dr S Sriram or Dr M Bhaskaran, Functional Materials and Microsystems Research Group, School of Electrical and Computer Engineering, RMIT University, GPO Box 2476, Melbourne, Victoria 3001, Australia. 
donor to a host substrate, our process offers a stronger bond to the PDMS substrate as well as easy processing with no need for tailored etching methods or dissolving of sacrificial layers. Such a ubiquitous process for transfer of functional, high-temperature-processed thin films with acid-free processing to elastomeric substrates will pave the way for touch sensing and energy harvesting for displays and electronics with flexible and transparent characteristics.

\section{EXPERIMENAL PROCEDURE}

\section{Transfer process for oxide to PDMS}

A silicon (100) wafer pre-cleaned with solvent and dried with compressed nitrogen was used as the supporting substrate for the process. Platinum and ITO thin films were deposited by electron beam evaporation at room temperature after pumping down to a base pressure of $1 \times 10^{-7}$ Torr. Both materials were deposited from high purity materials with platinum of $99.99 \%$ purity and a $99.95 \%$ purity mixture of $90 \%$ indium oxide and $10 \%$ tin oxide. Annealing of the ITO to render it transparent was performed in air at $400{ }^{\circ} \mathrm{C}$ for $1 \mathrm{~h}$ on establishing optimum resistivity values for the thin films. ${ }^{20}$ Removal of platinum by ion etching was performed using an argon plasma with a working pressure of $90 \mathrm{mTorr}$ and a radio frequency power of $100 \mathrm{~W}$ for 30 min. The transfer of the $\mathrm{ZnO}$ layer was similarly performed. The silicon wafer was cleaned and coated with platinum as described above. Subsequently, a $\mathrm{ZnO}$ layer was sputtered onto the platinum-coated wafer with the substrate temperature kept at $250{ }^{\circ} \mathrm{C}$ and a $6: 4$ gas ratio of $\mathrm{Ar} / \mathrm{O}_{2}$ with a radio frequency power of $200 \mathrm{~W}$. The removal of the platinum layer was performed by ion etching with the same parameters.

\section{Transmission measurements}

Ultraviolet and visible measurements were performed using a Cary 50 Bio spectrometer (Varian, Mulgrave, Victoria, Australia) over a $300-800 \mathrm{~nm}$ wavelength range and with a spot size of $1.0 \times 1.5 \mathrm{~mm}$.

\section{Uniaxial strain and endurance tests}

A custom-designed stretching stage was utilised. The samples under test were clamped between Perspex plates, with one end held on a fixed stage and the other end attached to a micrometre stage. The micrometre stage was controlled by a stepper motor (PD-110-42, Trinamic, Hamburg, Germany) with the aid of a RS-232 interface and custom-programmed LabView control.

\section{Scanning electron microscopy}

Scanning electron micrographs were collected using three different equipment configurations based on the information desired. The equipment used included: (i) a field emission gun scanning electron microscope (Nova NanoSEM, FEI Systems, Hillsboro, OR, USA) in a low vacuum mode. The samples were mounted orthogonally and tilted by $15^{\circ}$ to acquire images as viewed at $75^{\circ}$ from the surface normal. (ii) A FEI Quanta 200 ESEM in low vacuum mode. (iii) Imaging of stretching with a custom-made stretching apparatus capable of applying displacements with an accuracy of sub-10 $\mu \mathrm{m}$ steps.

\section{RESULTS}

Process for transfer of functional oxides onto polymer substrates Microfabrication, thin film processing and photolithography techniques were combined to realise a process for the transfer of high-temperature functional oxides onto PDMS substrates. For the purpose of demonstrating the transfer technique, and establishing functionality, the process is described using ITO thin films. These thin films require annealing at temperatures $>400{ }^{\circ} \mathrm{C}$, which is beyond polymer limits, to render them conductive and transparent. A schematic of the acid-free process to transfer high-temperature-processed oxides (or materials) onto flexible PDMS substrates is shown in Figure 1.

A photolithographically defined photoresist pattern on a precleaned silicon wafer is coated with $50 \mathrm{~nm}$ of platinum followed by $200 \mathrm{~nm}$ of ITO (see Figure 1a). Following lift-off in an acetone bath (Figure 1b), the sample is annealed at $400{ }^{\circ} \mathrm{C}$ in air for $1 \mathrm{~h}$. This step stabilises the stoichiometry of ITO and renders it conductive and transparent (Figure 1c). A 1:10 mixture of the curing agent and prepolymer mixture of PDMS is spun onto the wafer surface and cured, following which the entire layer is peeled off. The use of the $50 \mathrm{~nm}$ platinum layer enables this step, as platinum has very poor adhesion to silicon (and usually requires chromium or titanium adhesion layers ${ }^{13,21}$ ). The lack of an adhesion layer ensures that the platinum with the oxide material on its surface is embedded in the cured PDMS layer (Figure 1d). The entire platinum-ITO-PDMS structure is flipped over and placed on a silicon wafer for the removal of the platinum layer by dry etching (Figure 1e; see Supplementary Information). Now, the ITO-PDMS structure can be released to

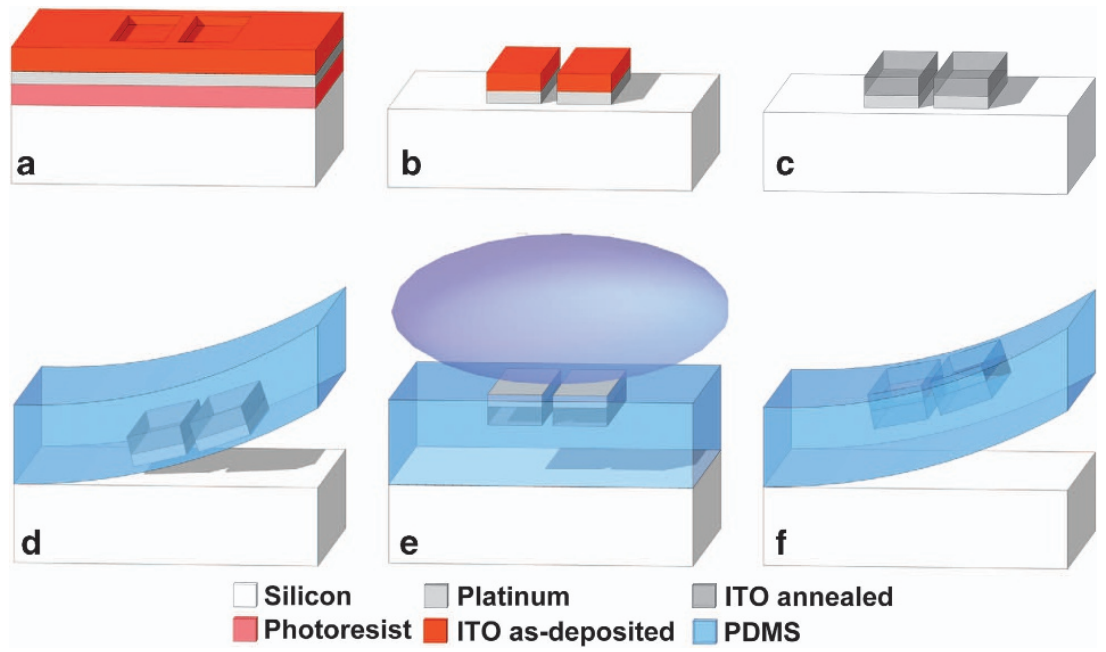

Figure 1 Schematic of process to transfer a high-temperature-processed oxide thin film on to a flexible polydimethylsiloxane (PDMS) substrate (demonstrated using indium tin oxide (ITO)). (a) A silicon substrate is photolithographically defined with a lift-off pattern followed by deposition of platinum and ITO layers. (b) Lift-off in solvents is completed to define ITO stripes for resistance measurements. (c) The as-deposited ITO is annealed at $400{ }^{\circ} \mathrm{C}$ to render it transparent. (d) PDMS is spin-coated and cured, following which the entire structure can be peeled off. (e) The released structure is flipped over and placed on silicon for removal of the platinum layer by plasma-assisted dry etching. (f) The final structure is peeled off to realize a flexible substrate embedded with high-temperature-processed oxide. 
realise a fully transparent and flexible substrate (Figure 1f), the functionality of which is defined by the first photolithography step (Figure 1a). Considering the proposed novelty of this process to transfer high-temperature oxides on to a flexible substrate, the transfer of high-temperature-deposited $\mathrm{ZnO}$ thin films was also studied and demonstrated (discussed later). Apart from the deposition of the $\mathrm{ZnO}$ thin film with a thickness of $450 \mathrm{~nm}$, through radio frequency magnetron sputtering at $250{ }^{\circ} \mathrm{C}$, no process parameters where changed.

\section{Transfer of micron-scale features}

With the capability to transfer large-area high-temperature-processed functional oxides, the ability to transfer of high-resolution features or patterns utilising the proposed transfer process was explored. A photolithography mask with sub- $10 \mu \mathrm{m}$ longitudinal stripes in close proximity was used, resulting in gaps after lift-off, as shown in Figure 2a. After the successive annealing step shown in Figure 2b, the pattern was transferred and the platinum layer removed. The results showed that consistent and good resolution could be achieved, as shown in Figure 2c (also see Supplementary Information). Furthermore, control over the crack formation in the ITO film could be gained. The random cracking of the sheet film, extensively discussed in the Results section, was suppressed by the longitudinal gaps in the ITO film with the cracks now occurring only in the direction of peeling off the PDMS for release from the substrate.

\section{Transmission measurements}

The level of transparency of the ITO-PDMS devices was quantitatively characterised by ultraviolet and visible spectroscopy. Identical ITO thin films were prepared on glass slides for comparison, with the transmission characteristics presented in Figure 3a. The results for the ITO thin films on glass matched the reported values. ${ }^{20}$ The results show that the ITO-PDMS devices transmit $>60 \%$ and up to $80 \%$ of the incident light across the visible range and at higher wavelengths. The limited visual impact of the ITO-PDMS combination over ITOglass is evident from the insets in Figure 3a, where a logo viewed through the ITO-PDMS is only marginally dull. The flexibility and transparency of the substrates realised is also illustrated photographically by Figure $3 b$.

\section{Strain-resistance measurements}

PDMS is utilised extensively for the realisation of flexible electronics because of its conformal, biocompatible, transparent and low surface energy properties. ${ }^{16,22}$ Resistors defined by gold and silver thin films on PDMS have been previously examined to determine the maximum strain before irreversible open circuit. Maximum strain values of $10 \%$ for gold resistors on as-formed $\mathrm{PDMS}^{23}$ and $20 \%$ or greater for pretreated and pre-stretched PDMS layers ${ }^{24}$ have been reported.

Utilising the process described by Figure 1, large area transparent conductive oxide ITO resistors of dimensions $1 \mathrm{~cm}$ long, $2 \mathrm{~mm}$ wide and $200 \mathrm{~nm}$ thickness were fabricated. The ability to realise such large area patterns, which are transparent, is a significant requirement for future technologies such as flexible displays or photovoltaic devices.
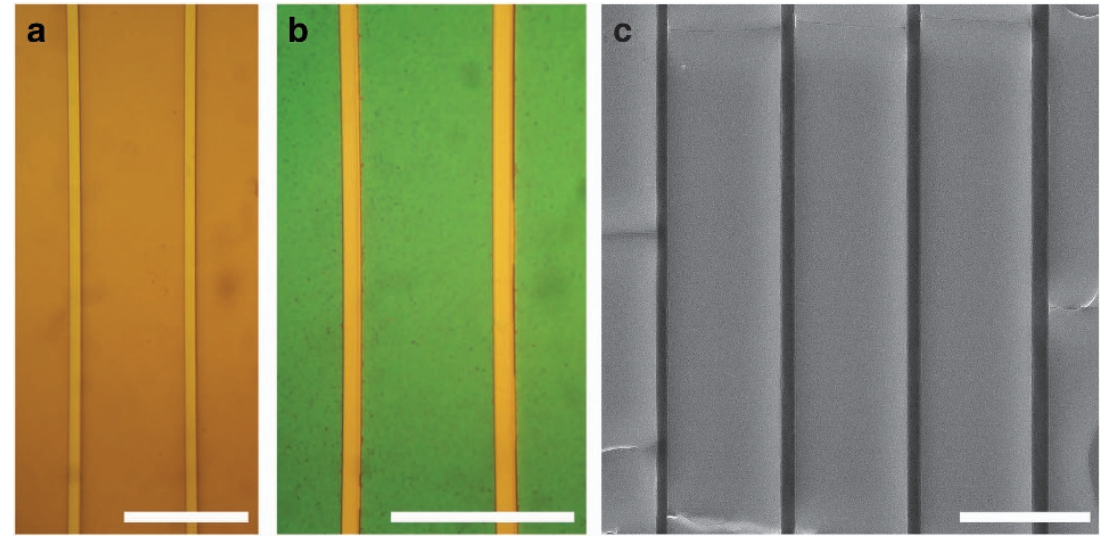

Figure 2 Transfer of high-resolution patterns of indium tin oxide (ITO) on to polydimethylsiloxane (PDMS). Patterned sub-10 $\mu \mathrm{m}$ gaps on Si before, after annealing and transferred to PDMS. (a) Optical micrograph of the patterned ITO-platinum film after lift-off. (b) Optical micrograph of the patterned ITO-platinum film after annealing. (c) Electron micrograph of the transferred ITO layer after dry etching the platinum layer. All scale bars are $50 \mu \mathrm{m}$.
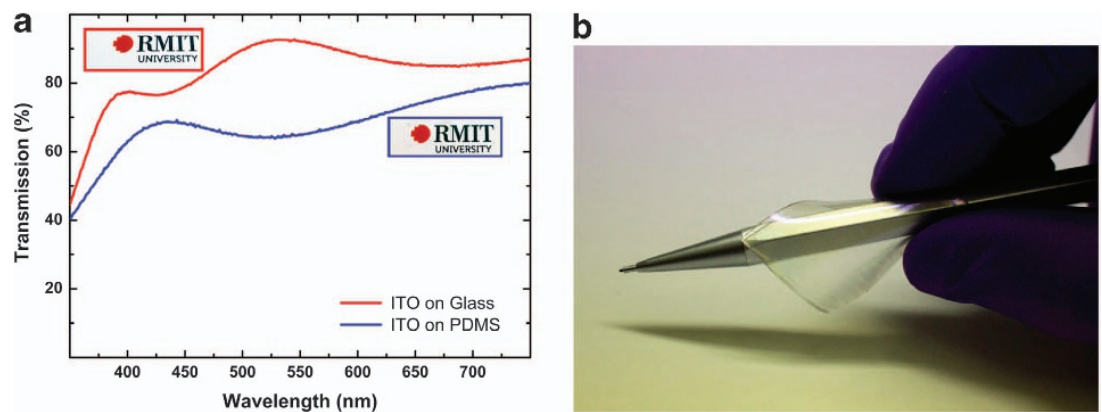

Figure 3 Evaluation of transparency of indium tin oxide (ITO)-polydimethylsiloxane (PDMS) sample. (a) Broadband transmission spectra for ITO-PDMS samples compared with ITO-glass depicting $>60 \%$ transmission in the visible region (evidenced by insets of samples placed on a logo). (b) A photograph of an ITO-PDMS sample on a pencil conveying both transparent and flexible characteristics. 


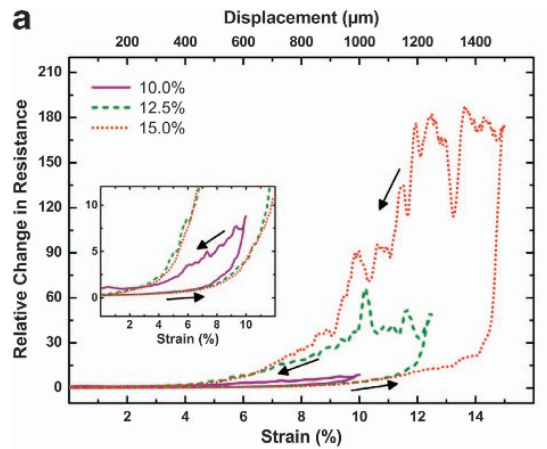

b

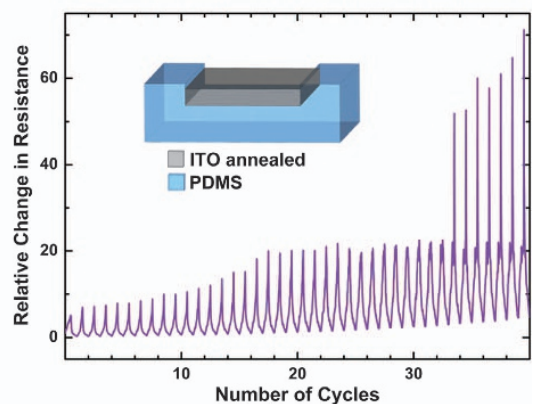

Figure 4 Uniaxial strain tests of indium tin oxide (ITO) on polydimethylsiloxane (PDMS). (a) ITO thin film patterns of $1 \mathrm{~cm}$ length and $2 \mathrm{~mm}$ width studied under different strain levels exhibit a maximum strain limit of 15\%, with minimal resistance degradation for strains of 10\% and lower. The inset in (a) highlights the very small resistance change for $10 \%$ strain cycles. (b) Reversibility strain-resistance tests performed over 40 cycles at $5 \%$ strain showed complete recoverability of properties albeit with marginal increase in peak strain resistance values. The inset in (b) shows a cross-section schematic of the tested sample.
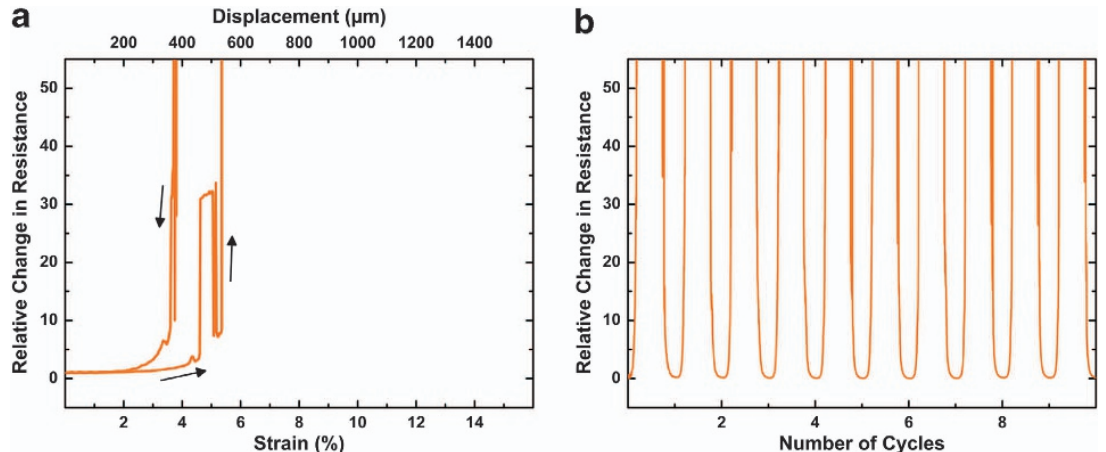

Figure 5 Uniaxial strain tests of gold on polydimethylsiloxane (PDMS). (a) The gold thin film resistors studied up to $10 \%$ strain fail at $\sim 5 \%$ because of their size, but can recover to original resistance state. (b) Reversibility strain-resistance tests of the gold-PDMS resistors over 10 cycles at $10 \%$ show recoverability but highlight dramatic resistance increases.

These linear resistor structures were subjected to precise uniaxial strain along their length controlled by a programmable motor and micrometre stage. The subsequent change in resistance relative to the initial resistance was studied as a function of applied strain.

The strain-resistance tests demonstrated that the ITO-PDMS resistors easily withstood strains of up to $10 \%$ with marginal change in the resistance values and were fully reversible to their original resistance state (inset of Figure 4a). At higher values of strains such as 12.5 and $15.0 \%$, the ITO-PDMS resistors showed respective increases of about $50 \times$ and $180 \times$ the initial resistance. Favourably, full reversibility to the initial resistance state was achieved with expected hysteresis behaviour ${ }^{25}$ displayed in the recovery process (Figure 4a).

The ITO-PDMS resistors were also subject to endurance tests under uniaxial strain. The resistors showed complete reversibility to their original resistance state for up to 30 cycles (Figure $4 \mathrm{~b}$ ). Beyond those cycles, a marginal increase in the zero strain resistance was apparent, and represents scope for future improvements and optimisation. It should also be noted that the maximum resistance value at peak strain gradually increased (often related to a priming process in flexible devices ${ }^{26}$ ), and was stable up to the 30-cycle mark. Although the 30-cycle endurance might appear as a limiting factor (and was the case for early gold-PDMS electrodes), this is a significant mark considering the process novelty, with significant room for improvements with design strategies such as curved or meandering electrodes.

Gold resistors were fabricated such that they were identical in geometry to the ITO-PDMS resistors presented in Figure 4. The fabrication process used involved standard photolithography on cured PDMS on silicon. ${ }^{6,7}$ Uniaxial strain was applied along the length of the gold-PDMS resistors, again identical in manner to the tests performed on the ITO-PDMS resistors. The results show the limited strain durability of these resistors as they experience dramatic increase in resistance for strains above $4.7 \%$ (Figure 5). Such strain resistance measurements performed over 10 cycles also show systematic open circuit at $4.7 \%$ on average, but exhibit full recovery, in conformance with the theory of gold micro-islands enabling conductivity and reversibility. $^{27}$

\section{DISCUSSION}

In proposing a novel technique for the realisation of high-temperature-processed oxide thin films on flexible substrates, our work utilised ITO and $\mathrm{ZnO}$ on PDMS for demonstration. The strainresistance relationship relevant to flexible and stretchable electronics highlighted that the ITO-PDMS structures endure greater strain $(15 \%)$ than their identically sized gold-PDMS $(<10 \%)$ counterparts. Although a favourable result, this is unexpected when considering a brittle oxide material in relationship to gold thin films, which are metallic and known to be malleable. ${ }^{23}$

In order to understand the surface microstructure of the ITO on PDMS, and related factors that would enable greater strain durability in an oxide material, the fabricated samples were imaged using scanning electron microscopy. The scanning electron micrograph of an ITO-PDMS sample resulting from Figure if is 
shown in Figure 6a. The micrograph shows that the micro-cracking in ITO results in plate-like structures with overlapping edges. It should be noted that the micrograph is collected at an exaggerated angle of $75^{\circ}$ from the surface normal to enable visualisation of the plate-like structure. The surface microstructure shows minimal change following multiple stretching cycles, with the micrograph for a sample stretched 50 times shown in Figure $6 \mathrm{~b}$ appearing similar to Figure 6a. There is also no evidence of any delamination.
This led to the hypothesis that the ITO plates behave like geological tectonic plates that are capable of sliding over each other to attain equilibrium states. The hypothesis is schematically depicted in Figure $6 c$, where, on stretching, the ability of the plates to slide over each other ensures conductivity is retained for higher levels of strain.

To test this hypothesis, a custom-made stretching stage with the ability to work in a scanning electron microscope was designed and, in situ images of stretched ITO on PDMS were collected. The insights gained correlate with the resistive measurements. Figure 7a shows that
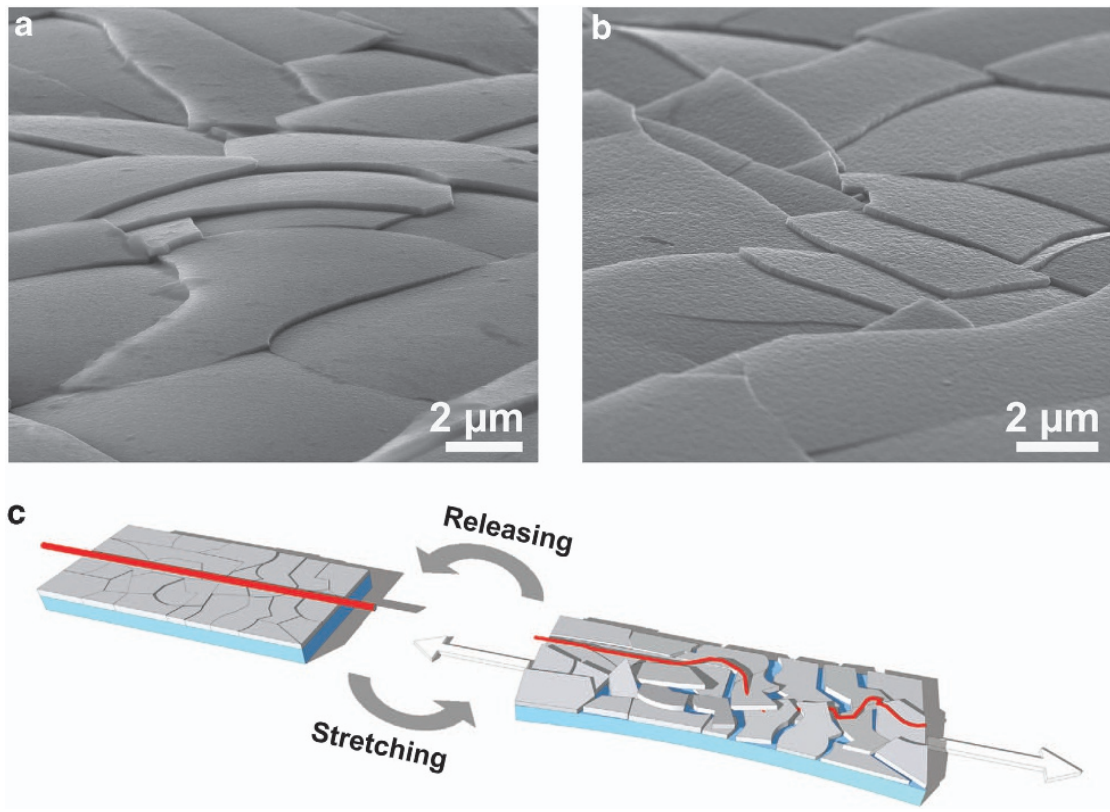

Figure 6 Micro-tectonics enabled high strain performance. (a, b) Scanning electron micrographs at $75^{\circ}$ tilt depicting plate-like oxide surface structure in indium tin oxide (ITO)-polydimethylsiloxane (PDMS) samples as-fabricated and after multiple cycles of stretching, respectively. (c) Schematic depiction of the path taken by electrical current (red line) through the ITO plates capable of sliding over each other to retain electrical conductivity contributing to greater strain performance.

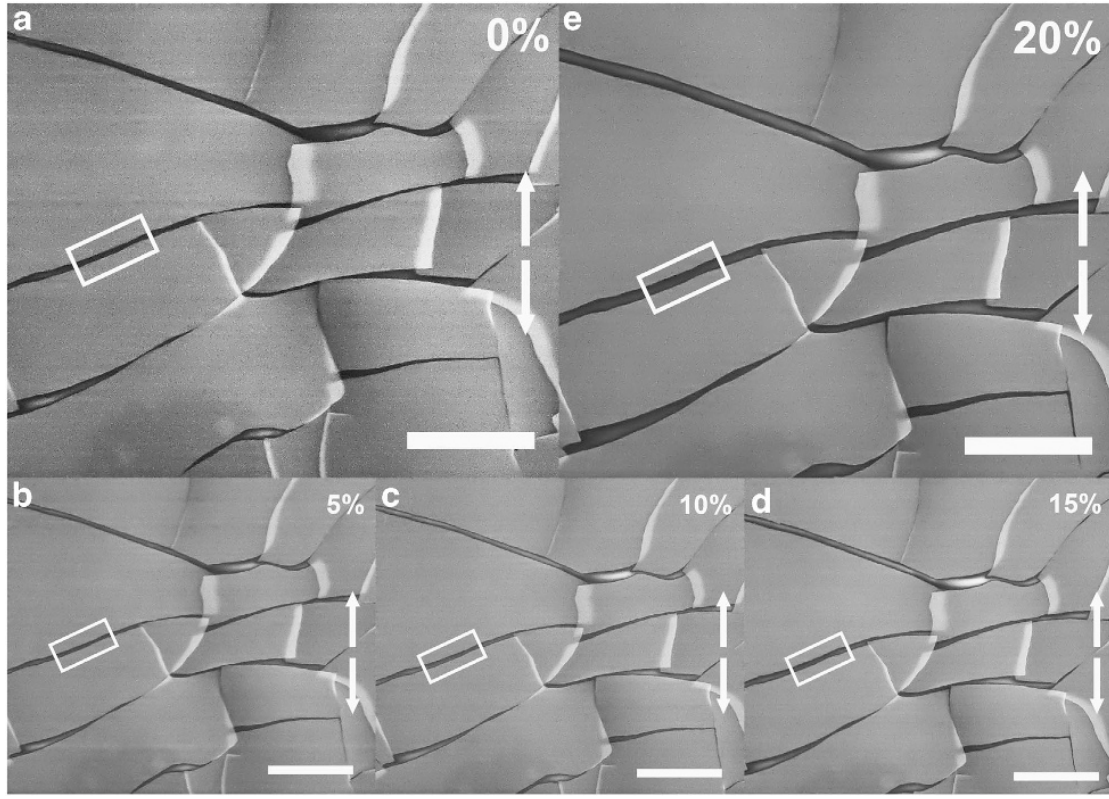

Figure 7 Observation of micro-tectonic plates under different levels of strain. Strains from 0 to $20 \%$ in steps of $5 \%$ are shown in (a)-(e). The scale bar in all pictures is $10 \mu \mathrm{m}$ in length. The marked region shows a representative gap between the micro-tectonic plates. 

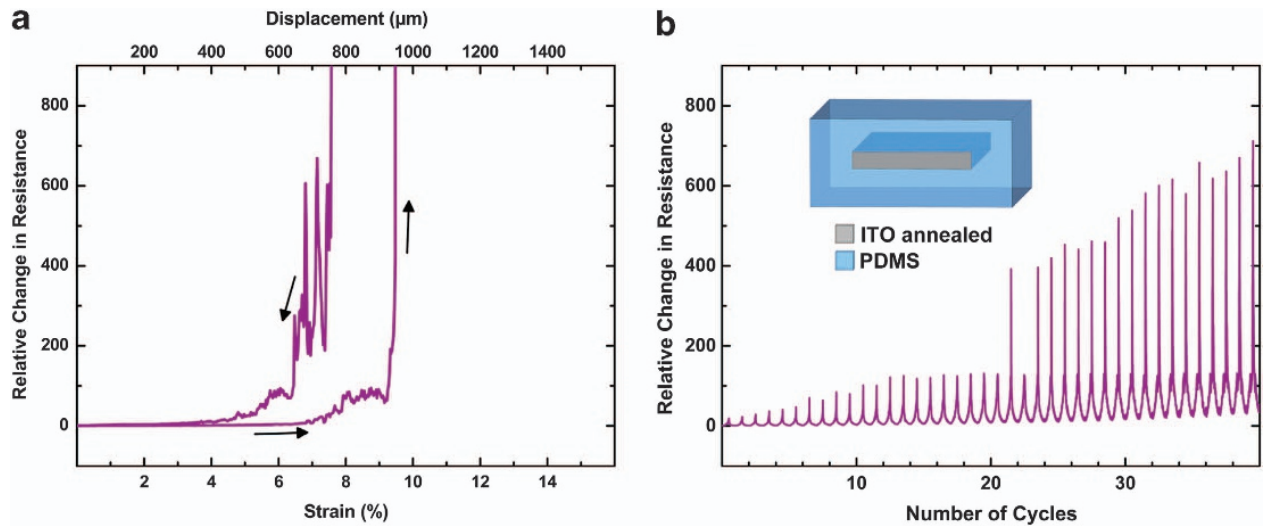

Figure 8 Uniaxial strain tests of encapsulated indium tin oxide (ITO) on polydimethylsiloxane (PDMS). (a) ITO thin film resistors with encapsulation experience complete open circuit at $10 \%$ strain, but can recover to original resistance state. The resistance ratio at $7.5 \%$ strain exceeds 25 , which is three times higher than non-encapsulated samples. (b) Reversibility strain-resistance tests performed over 40 cycles at $5 \%$ strain showed complete recoverability, however, changes in resistances are 100-200 times higher with encapsulation. The inset in (b) shows a cross-section schematic of the tested sample.
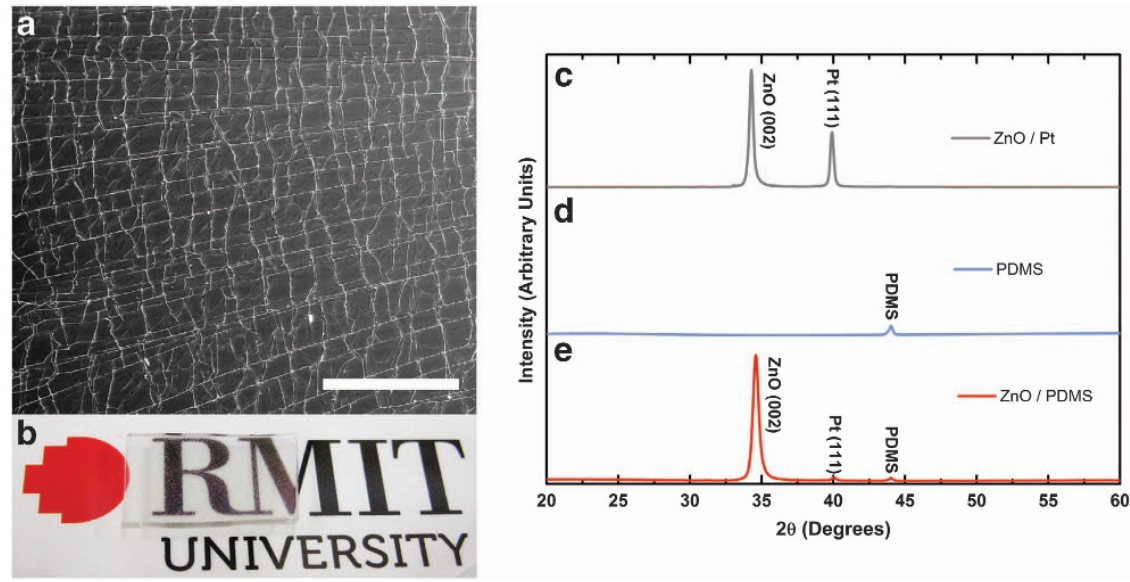

Figure 9 (a) Scanning electron micrograph of the transferred zinc oxide (ZnO; scale bar $500 \mu \mathrm{m}$ ). (b) Transparent slab of polydimethylsiloxane (PDMS)coated $\mathrm{ZnO}$. X-ray diffractograms of: (c) deposited $\mathrm{ZnO}$ on platinum before transfer, (d) Bare PDMS slab and (e) ZnO after transfer and removal of platinum.

in un-stretched ITO, as expected all the micro-tectonic plates are in contact. With increasing strain, the plates slowly drift away from each other and the overlapping area decreases as seen in Figures $7 \mathrm{~b}$ and $\mathrm{c}$. The visible change is not very significant (see the marked area), which is expected after observing that the change in resistance is not severe at this level of strain. On applying $>10 \%$ strain, the micro-tectonic plate movement becomes more pronounced as can be seen in Figures $7 \mathrm{~d}$ and e. In addition, the corresponding resistance measurement indicates a drastic change of up to $180 \%$. At $20 \%$ strain, the ITO film shows major gaps between the plates, which result in an open circuit when measuring resistance as shown in Figure 7e. This is unlike the accepted mechanism for gold where the micro-cracked islands slide against and not over each other. ${ }^{27}$ Furthermore, the good adhesion of ITO to PDMS and the micro-tectonics enables the plates to slide back on to each other allowing full recovery to the initial resistance state.

An effective method to test this hypothesis is to include an encapsulating layer of PDMS on top of the ITO, which would limit one degree of freedom. This can be considered a further extension of the tectonics analogy where constraining the movement of plates creates a build up of pressure. ITO-PDMS resistors identical to those presented in Figure 4 were prepared with an additional encapsulating PDMS layer. This creates a PDMS-ITO-PDMS sandwich structure, which restricts the freedom of movement of the ITO plates.
These resistors were studied under applied uniaxial strain with results presented in Figure 8. Change in resistance values in the stretching cycle are over three times higher for strains $>7 \%$ in comparison with the non-encapsulated samples (inset of Figure 4a). At $10 \%$ maximum strain the resistor transforms from the very high resistance state $(>7 \%$ strain) to open circuit states, but with recoverability of the initial resistance. These samples also exhibit reversible performance over multiple cycles, with a directly comparable experiment to Figure $4 \mathrm{~b}$ presented in Figure $8 \mathrm{~b}$. The significant observation is that the change in resistance is two orders of magnitude higher for the encapsulated samples. This allows the conclusion that encapsulation in the case of ITO-PDMS significantly degrades performance, in stark contrast to the gold-PDMS resistors. ${ }^{25,26}$

This result highlights the significance of the added degree of freedom required for the plates to slide across each other and retain conductivity. This significant microscale phenomenon contributes to the enhanced strain-resistance performance, but does not degrade the macroscale transmission characteristics (Figure 3a). A recent study conducted by Chae et al. ${ }^{28}$ demonstrated that it is not significant for thin films to be perfectly planar to show high performance. Therefore, we believe that our technology to transfer high-temperature oxides provides a platform for many future applications. 
By comparison, it is known that for gold on PDMS structures, the large differences in thermal expansion coefficients result in cracks in the material, which affect adhesion and durability. ${ }^{25}$ For this reason, successful gold electrode structures are encapsulated an additional layer of PDMS to prevent delamination, with such encapsulation enabling $\sim 12 \%$ strain before failure. ${ }^{24-26}$

In addition to the strain experiments, we investigated an additional oxide thin film, to verify if micro-tectonic plates occur in different oxide films with various thicknesses. Furthermore, the crystal structure was studied before and after the transfer to verify crystallinity. The transferred $450 \mathrm{~nm}$ thick transparent $\mathrm{ZnO}$ layers, see Figure 9a, show similar behaviour to the $200 \mathrm{~nm}$ thick ITO. The oxide film is cracked during the release from the silicon carrier substrate, as seen in the electron micrograph in Figure 9b. The micro-tectonic plates are similar in size to those of ITO. X-ray diffractograms show that the as-deposited $\mathrm{ZnO}$ is highly crystalline (Figure 9c). The transferred $\mathrm{ZnO}$ layers on PDMS were also characterised by X-ray diffractograms, and compared with a bare PDMS reference (Figure 9d). The crystallinity of the transferred $\mathrm{ZnO}$ remains intact (Figure 9e) and is directly comparable to the as-deposited $\mathrm{ZnO}$ (Figure 9c) with removal of the platinum by ion etching evident.

\section{CONCLUSION}

We present a novel process for high-temperature-processed oxide thin films on to polymeric substrates. The process demonstrated with the transparent conductor ITO on the flexible elastomer PDMS demonstrates high strain-resistance performance, with significant strain limits of $15 \%$ compared with gold on PDMS $(<10 \%)$. An overlapping plate-like thin film microstructure contributes to the enhanced strain performance; where this unique microstructure offers specific advantages that complement thin film electrodes. We demonstrate excellent patterning ability with sub$10 \mu \mathrm{m}$ features and versatility of the process through transfer of hightemperature-deposited $\mathrm{ZnO}$. We consider that the acid-free and scalable transfer process presented for integration of high-temperature-processed functional materials onto flexible substrates will enable a plethora of applications, in addition to creating opportunities for discoveries such as the influence of micro-tectonics on strainresistance performance.

\section{CONFLICT OF INTEREST}

The authors declare no conflict of interest.

\section{ACKNOWLEDGEMENTS}

PG acknowledges an Australian Government Endeavour International Postgraduate Research Scholarship. SS and MB acknowledge Australian PostDoctoral Fellowships from the Australian Research Council through Discovery Projects DP110100262 and DP1092717, respectively. They acknowledge the facilities and technical assistance of the Australian Microscopy and Microanalysis Research Facility at the RMIT Microscopy and Microanalysis Facility at RMIT University.

1 Carlson, A., Bowen, A. M., Huang, Y., Nuzzo, R. G. \& Rogers, J. A. Transfer printing techniques for materials assembly and micro/nanodevice fabrication. Adv. Mater. 24, 5284-5318 (2012).

2 Huang, F. \& Baumberg, J. J. Actively-tuned plasmons on elastomerically-driven Au nanoparticle dimers. Nano Lett. 10, 1787-1792 (2010).

3 Kim, B. J., Jang, H., Lee, S.-K., Hong, B. H., Ahn, J.-H. \& Cho, J. H. High-performance flexible graphene field effect transistors with ion gel gate dielectrics. Nano Lett. 10, 3464-3466 (2010)
4 Kim, R.-H., Kim, D.-H., Xiao, J., Kim, B. H., Park, S.-I., Panilaitis, B., Ghaffari, R., Yao, J., Li, M., Liu, Z., Malyarchuk, V., Kim, D. G., Le, A.-P., Nuzzo, R. G., Kaplan, D. L., Omenetto, F. G., Huang, Y., Kang, Z. \& Rogers, J. A. Waterproof AllnGaP optoelectronics on stretchable substrates with applications in biomedicine and robotics. Nat. Mater. 9, 929-937 (2010).

5 Qi, Y., Jafferis, N. T., Lyons, K., Lee, C. M., Ahmad, H. \& McAlpine, M. C. Piezoelectric ribbons printed onto rubber for flexible energy conversion. Nano Lett. 10, 524-528 (2010).

6 Shah, C. M., Sriram, S., Bhaskaran, M., Nasabi, M., Nguyen, T. G., Rowe, W. S. T. \& Mitchell, A. Elastomer-based pneumatic switch for radio frequency microdevices. J. Microelectromech. Sys. 21, 1410-1417 (2012).

7 Khodasevych, I. E., Shah, C. M., Sriram, S., Bhaskaran, M., Withayachumnankul, W., Ung, B. S. Y., Lin, H., Rowe, W. S. T., Abbott, D. \& Mitchell, A. Elastomeric silicone substrates for terahertz fishnet metamaterials. Appl. Phys. Lett. 100, 061101 (2012)

8 Sun, L., Qin, G., Huang, H., Zhou, H., Behdad, N., Zhou, W. \& Ma, Z. Flexible highfrequency microwave inductors and capacitors integrated on a polyethylene terephthalate substrate. Appl. Phys. Lett. 96, 013509 (2010).

9 Takei, K., Takahashi, T., Ho, J. C., Ko, H., Gillies, A. G., Leu, P. W., Fearing, R. S. \& Javey, A. Nanowire active-matrix circuitry for low-voltage macroscale artificial skin. Nat. Mater. 9, 821-826 (2010).

10 Nomura, K., Ohta, H., Takagi, A., Kamiya, T., Hirano, M. \& Hosono, H. Roomtemperature fabrication of transparent flexible thin-film transistors using amorphous oxide semiconductors. Nature 432, 488-492 (2004).

11 Lim, H., Cho, W. J., Ha, C. S., Ando, S., Kim, Y. K., Park, C. H. \& Lee, K. Flexible organic electroluminescent devices based on fluorine-containing colorless polyimide substrates. Adv. Mater. 14, 1275-1279 (2002).

12 Wang, Z. L. \& Wu, W. Nanotechnology - enabled energy harvesting for self-powered micro-/nanosystems. Angew. Chem. Int. Ed. 51, 11700-11721 (2012).

13 Bhaskaran, M., Sriram, S., Mitchell, D. R. G., Short, K. T. \& Holland, A. S. Effect of multi-layered bottom electrodes on the orientation of strontiumdoped lead zirconate titanate thin films. Thin Solid Films 516, 8101-8105 (2008).

14 Dai, Z. R., Pan, Z. W. \& Wang, Z. L. Novel nanostructures of functional oxides synthesised by thermal evaporation. Adv. Funct. Mater. 13, 9-24 (2003).

15 Khartsev, S. I., Grishin, M. A. \& Grishin, A. M. Characterization of heteroepitaxial $\mathrm{Na}_{0.5} \mathrm{~K}_{0.5} \mathrm{NbO}_{3} / \mathrm{La}_{0.5} \mathrm{Sr}_{0.5} \mathrm{CoO}_{3}$ electro-optical cell. Appl. Phys. Lett. 86, 062901 (2005).

16 Rogers, J. A. \& Nuzzo, R. G. Recent progress in soft lithography. Mater. Today 8, 50-56 (2005).

17 Romanowsky, M. B., Heymann, M., Abate, A. R., Krummel, A. T., Fraden, S. \& Weitz, D. A Functional patterning of PDMS microfluidic devices using integrated chemo- masks. Lab. Chip. 10, 1521-1524 (2010).

18 Ozgur, U., Alivov, Y. I., Liu, C., Teke, A., Reshchikov, M., Dogan, S., Avrutin, V., Cho, S.-J. \& Morkoc, H. A. A comprehensive review of $\mathrm{ZnO}$ materials and devices. J. Appl. Phys. 98, 041301 (2005).

19 Meitl, M. A., Zhu, Z.-T., Kumar, V., Lee, K. J., Feng, X., Huang, Y. Y., Adesida, I., Nuzzo, R. G. \& Rogers, J. A. Transfer printing by kinetic control of adhesion to an elastomeric stamp. Nat. Mater. 5, 33-38 (2005).

20 George, J. \& Menon, C. Electrical and optical properties of electron beam evaporated ITO thin films. Surf. Coat. Technol. 132, 45-48 (2000).

21 Tiggelaar, R. M., Sanders, R. G. P., Groenland, A. W. \& Gardeniers, J. G. E. Stability of thin platinum films implemented in high-temperature microdevices. Sens. Actuators, $A$ 152, 39-47 (2009).

22 Xia, Y. \& Whitesides, G. M. Soft lithography. Annu. Rev. Mater. Sci. 28, 153-184 (1998).

23 Lacour, S. P., Wagner, S., Huang, Z. \& Suo, Z. Stretchable gold conductors on elastomeric substrates. Appl. Phys. Lett. 82, 2404-2406 (2003).

24 Lacour, S., Jones, J., Suo, Z. \& Wagner, S. Design and performance of thin metal film interconnects for skin-like electronic circuits. IEEE Electron. Dev. Lett. 25, 179-181 (2004).

25 Durnin, B. M., Shah, C. M., Sriram, S. \& Bhaskaran, M. Strain-resistance relationship in gold conductors for elastomeric-based flexible devices. Proc. SPIE 8204, 820437 (2011).

26 Lacour, S., Chan, D., Wagner, S., Li, T. \& Suo, Z. Mechanisms of reversible stretchability of thin metal films on elastomeric substrates. Appl. Phys. Lett. 88, 204103 (2006)

$27 \mathrm{Graz}$, I., Cotton, D. \& Lacour, S. Extended cyclic uniaxial loading of stretchable gold thin-films on elastomeric substrates. Appl. Phys. Lett. 94, 071902 (2009).

28 Chae, S. H., Yu, W. J., Bae, J. J., Duong, D. L., Perello, D., Jeong, H. Y., Ta, Q. H., Ly, T. H., Vu, Q. A. \& Yun, M. Transferred wrinkled $\mathrm{Al}_{2} \mathrm{O}_{3}$ for highly stretchable and transparent graphene-carbon nanotube transistors. Nat. Mater. 12, 403-409 (2013).

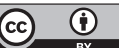

This work is licensed under a Creative Commons Attribution 3.0 Unported License. To view a copy of this license, visit http://creativecommons.org/licenses/by/3.0/

Supplementary Information accompanies the paper on the NPG Asia Materials website (http://www.nature.com/am) 